\title{
ON THE CLASSIFICATION OF KNOTS
}

\author{
KENNETH A. PERKO, JR.
}

\begin{abstract}
Linking numbers between branch curves of irregular covering spaces of knots are used to extend the classification of knots through ten crossings and to show that the only amphicheirals in Reidemeister's table are the seven identified by Tait in 1884. Diagrams of the 165 prime 10-crossing knot types are appended. (Murasugi and the author have proven them prime; Conway claims proof that the tables are complete.) Including the trivial type, there are precisely 250 prime knots with ten or fewer crossings.
\end{abstract}

This paper completes the classification (by knot type) of the 10-crossing knots tabled by Tait [9] and Little [4] and solves the amphicheirality problem for the six remaining knots in Reidemeister's table [6, pp. 70-72].

The method of proof is that set forth in [6, Chapter III, $\$ 15]$-linking numbers between branch curves of irregular covering spaces. 1 See also [3, \$5 and Reference 13].

The coverings considered are those arising out of representations of a knot group onto the dihedral group of order $2 p$ (the group of symmetries of a regular $p$-gon) or the symmetric group on four letters. The latter are restricted to representations sending meridians into transpositions and $v_{4}$ is the linking number betwe en the branch curve of branching index 2 and the algebraic sum of those of index 1 . Similarly, $v_{p}$ ( $p$ odd) is the linking number between the curve of index 1 and the sum of those of index 2 in a $p$ sheeted dihedral covering and $v_{p}(x, y)$ is the linking number in such a covering between the sum of all curves of index 2 which have linking number $x$ with the curve of index 1 and the sum of all curves of index 2 which have linking number $y$ with the curve of index 1 .

Received by the editors July 3, 1973 and, in revised form, September 12, 1973. AMS (MOS) subject classifications (1970). Primary 55A25, 55A10.

Key words and phrases. Knots with ten crossings, amphicheirality, linking numbers between branch curves of irregular covering spaces of knots.

1 These linking numbers may be computed geometrically as discussed in the author's paper On covering spaces of knots, to appear in Glasnik Matematicki 9 (29) (1974). Calculations with respect to particul ar examples are available on request. 
Since the index of branching is preserved under autohomeomorphisms of a branched covering induced by autohomeomorphisms of $S^{3} \supset k$, such linking numbers (taken as a set where there is more than one representation) are topological invariants of the complement of $k$ in $S^{3}$ which also prove nonamphicheirality where the set of such numbers (for a particular orientation of $S^{3}$ and all representations onto a given group) is nonsymmetric with respect to a simultane ous reversal of sign.

Of the 84 knots in Reidemeister's table, $4_{1}, 6_{3}, 8_{3}, 8_{9}, 8_{12}, 8_{17}$ and $8_{18}$ are known to be amphicheiral [8] and all but the following six have previously been proven nonamphicheiral by various means, including the Minkowski units [6, p. 31], Schubert's theorems [7, p. 170], signatures [5, p. 400], linking numbers in 3-sheeted dihedral covering spaces [3, p. 200] and a representational invariant of Burde [1, p. 120]. (Note that Schubert's table of 2-bridged knots has been extended by Murasugi [unpublished] to include $9_{19}, 9_{23}$ and $9_{26^{\circ}}$ ) The linking numbers tabled below prove that all six are nonamphicheiral.

$$
\begin{aligned}
& 8_{20} \quad v_{9}= \pm 2, \quad 9_{30} \quad v_{53}= \pm 8, \quad 9_{41} \quad v_{7}= \pm 6 / 7 \quad \text { (8 times) } \\
& 9_{24} \quad v_{9}= \pm 2, \quad 9_{33} \quad v_{61}(6,-6)= \pm 4, \quad 9_{49} \quad v_{5}= \pm 16 / 5 \quad \text { (6 times). }
\end{aligned}
$$

Of the 166 10-crossing knots in the Tait-Little tables, all but 31 have been previously distinguished (both from each other and from the knots in Reidemeister's table) either by their Alexander polynomials or, in 3 cases, by the rational fractions associated with 2-bridged knots (and corresponding, we are told, to the parameters of the lens spaces which constitute their 2-sheeted cyclic coverings) [2]. Table 1 sets forth these examples, grouped by polynomial type, together with linking numbers which newly distinguish 30 of them. As shown in Figure 1 , the pair $10_{511} 10_{6 \mathrm{VI}}$ turns out to be a previously undetected duplication in Little's table.
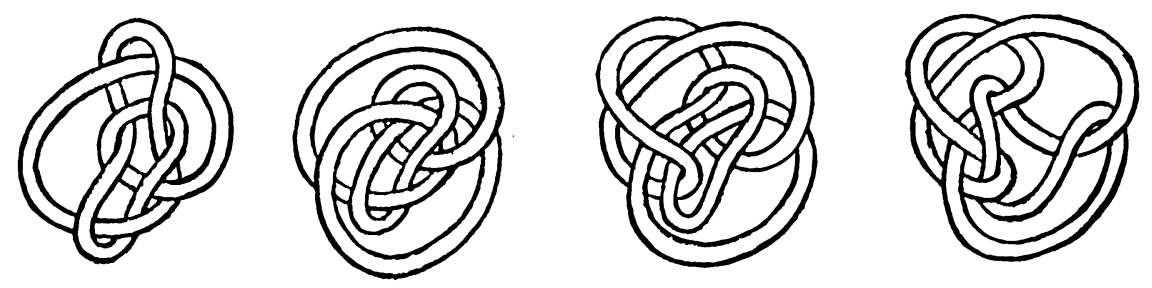

Figure 1 
Table 1

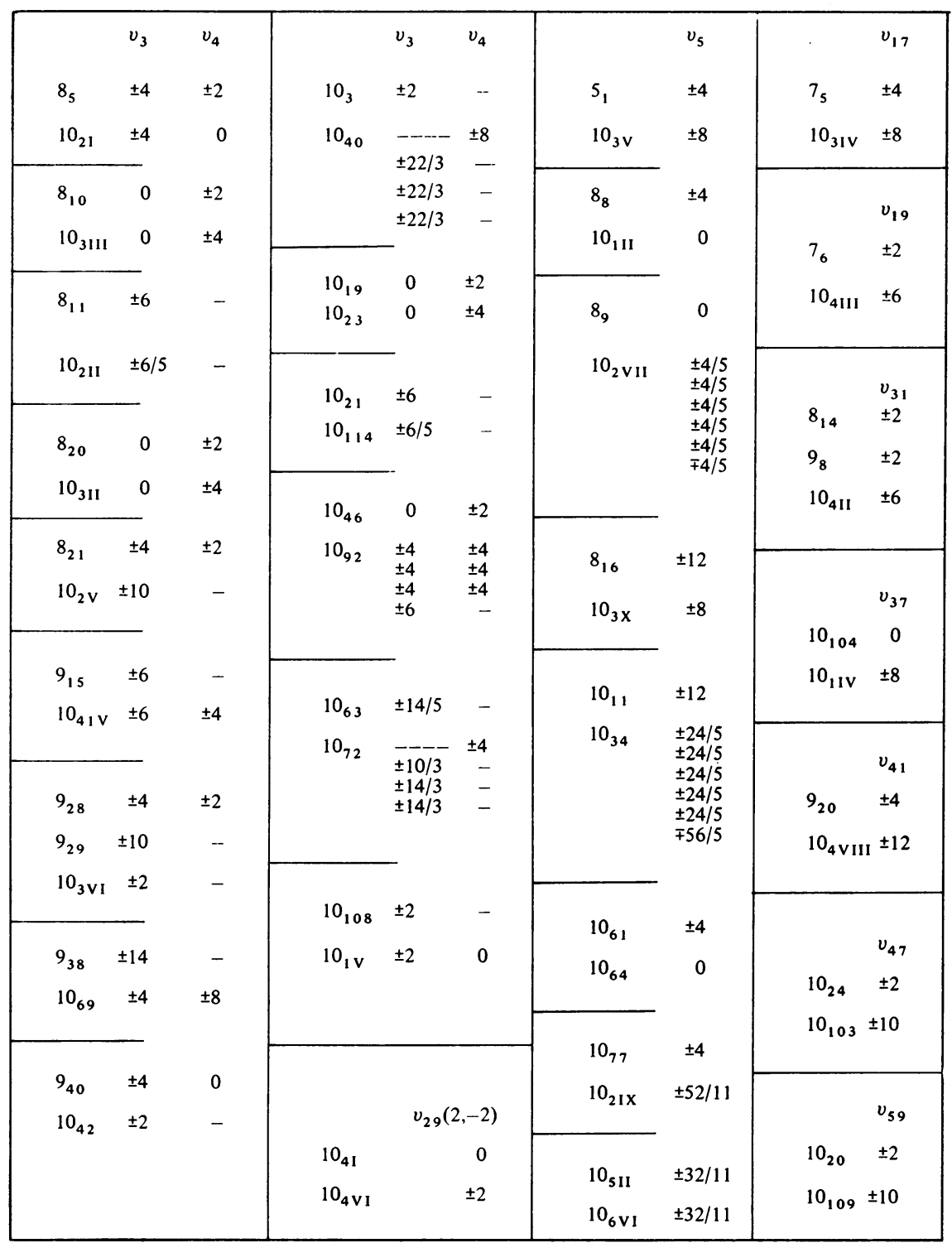




\section{PRIME KNOTS WITH TEN CROSSINGS}

Taken from the tables of P. G. Tait and C. N. Little [Trans. Roy. Soc. Edinburgh 32 (1885) and 39 (1900)] (The duplication in Little's table has been omitted and knots with two bridges are grouped at the beginning.)

$\$ 8 \%$

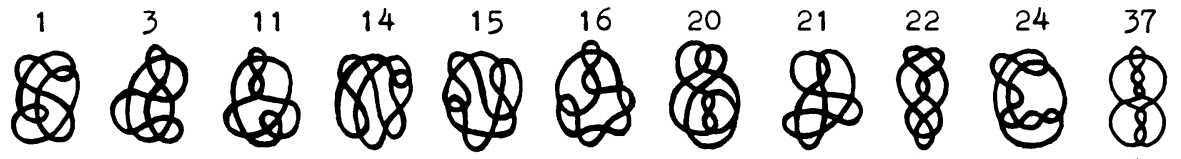

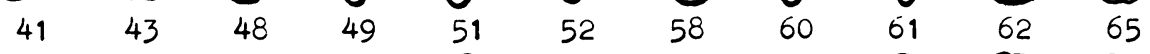

\& \& \& \& \& 818 (3)

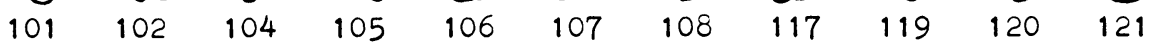

\&3 \& (8) ${ }_{122}{ }^{4}{ }^{5}{ }^{6}{ }_{8}{ }_{9}^{10}{ }_{12}^{13}$

(\$) \$2\% $\begin{array}{llllllllll}17 & 18 & 19 & 23 & 25 & 26 & 27 & 28 & 29 & 30\end{array}$

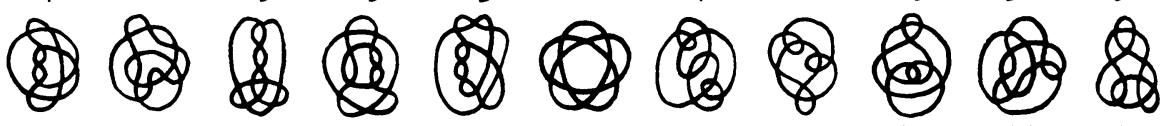

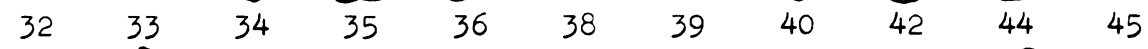

$\$ 28$

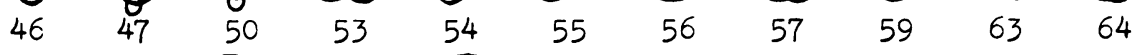

(3) 8

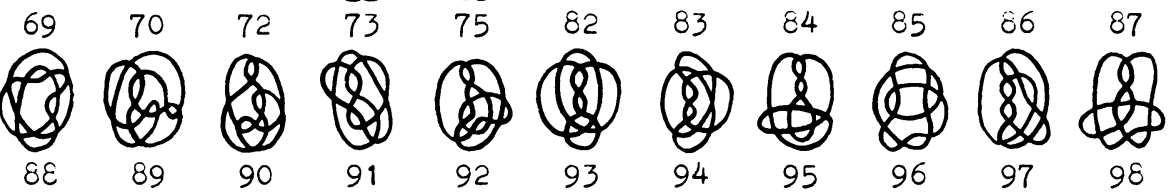




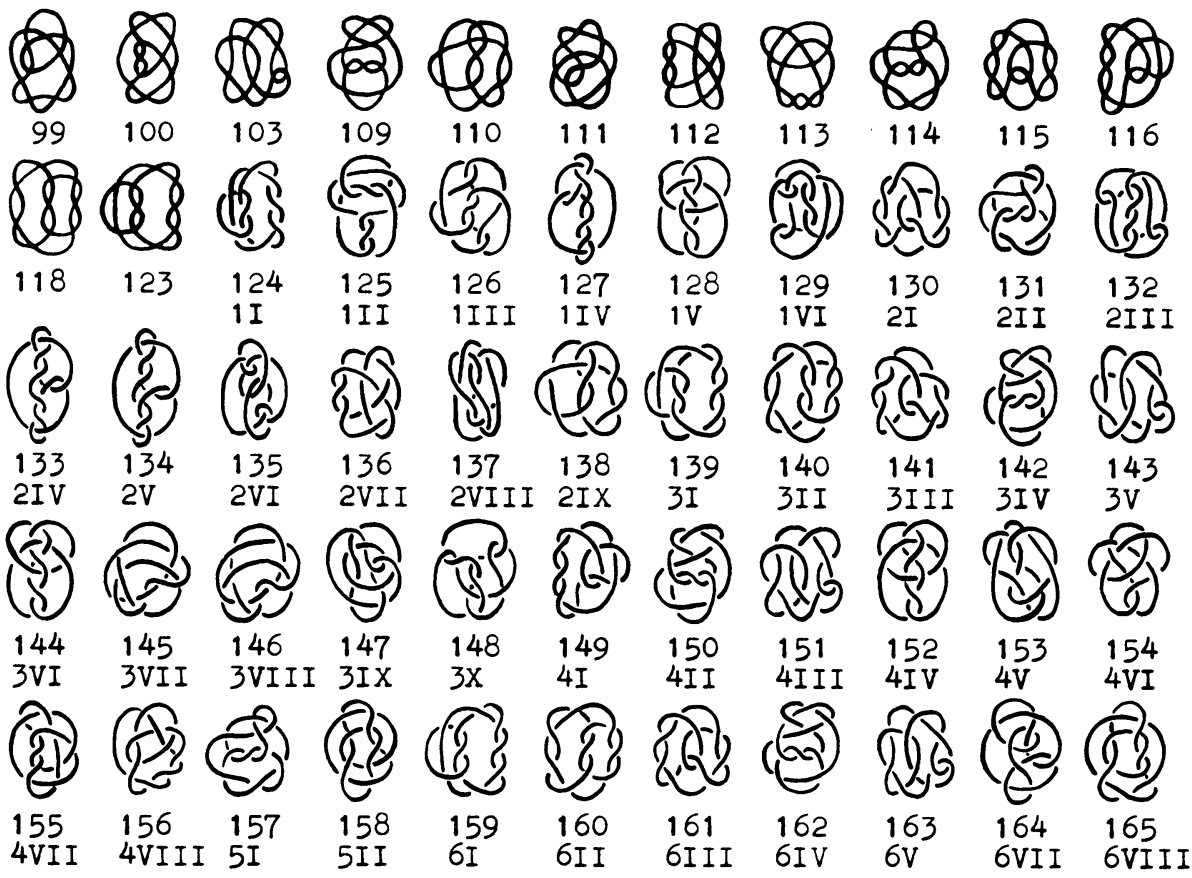

\section{REFERENCES}

1. G. Burde, Darstellungen von Knotengruppen und eine Knoteninvariante, Abh. Math. Sem. Univ. Hamburg 35 (1970), 107-120. MR 43 \#2695.

2. J.H. Conway, An enumeration of knots and links, and some of their algebraic properties, Computational Problems in Abstract Algebra (Proc. Conf., Oxford, 1967), Pergamon Press, Oxford, 1970, pp. 329-358. MR 41 \#2661.

3. R. H. Fox, Metacyclic invariants of knots and links, Canad. J. Math. 22 (1970), 193-201. MR 41\#6197.

4. C. N. Little, Non-alternate \pm knots, Trans. Roy. Soc. Edinburgh 39 (1900), $771-778$, pl ates I, II, III.

5. K. Murasugi, On a certain numerical invariant of link types, Trans. Amer. Math. Soc. 117 (1965), 387-422. MR 30 \#1506.

6. K. Reidemei ster, Knotentheorie, Ergebni sse der Math. und ihrer Grenzgebiete, Band 1, Springer-Verlag, Berlin, 1932.

7. H. Schubert, Knoten mit zwei Brücken, Math. Z. 65 (1956), 133-170. MR 18, 498.

8. P. G. Tait, The first seven orders of knottiness, Trans. Roy. Soc. Edinburgh 32 (1884), plate 44 .

9. - Tenfold knottiness, Trans. Roy. Soc. Edinburgh 32 (1885), plates 80,81 .

400 Central Park West Apt. 16-P, New York, New York 10025 